\title{
Multifidus Muscle Stiffness in Single-level Unilateral Lumbar Disc Herniation: Comparison of Two Shear-wave Elastography Methods
}

\author{
- Tuba Selcuk Can, • Behice Kaniye Yilmaz, @ Sevim Ozdemir \\ University of Health Sciences Turkey, Istanbul Haseki Training and Research Hospital, Clinic of Radiology, Istanbul, Turkey
}

Abstract

Aim: The suitability of shear-wave elastography (SWE) analysis in muscle tissue has been demonstrated previously. There are no studies that have applied the two SWE methods to evaluate the multifidus muscle in patients with lumbar disc herniation. We aimed to evaluate multifidus muscle stiffness in patients with single-level unilateral lumbar disc herniation via two SWE methods.

Methods: This cross-sectional study was carried out by examining the hospital records of patients who underwent lumbar MRI in July 2019. A total of 22 patients with single-level unilateral lumbar disc herniation underwent bilateral multifidus stiffness assessment via the two-dimensional SWE (2D-SWE) and point-SWE ( $p$-SWE) methods.

Results: The only measurement that demonstrated a significant difference between normoweight and overweight subjects was the hernia-side p-SWE value. On the hernia side, 2D-SWE and p-SWE were correlated. Age was determined to be an independent factor that significantly altered results for the hernia and non-hernia sides of both techniques, while weight was an independent factor for the hernia and non-hernia results of only the p-SWE technique.

Conclusion: Lower muscle stiffness determined via 2D-SWE or p-SWE may have value in the diagnosis, follow-up, or management of patients with one-sided unilateral lumbar disc herniation. Age and weight appear to be important variables to consider when evaluating multifidus muscle stiffness values.

Keywords: Body mass index, height, multifidus muscle, shear-wave elastography, weight

\section{Introduction}

Chronic low back pain is a common condition since it may be associated with a variety of underlying pathologies, including lumbar disc herniation, facet joint degeneration, paraspinal muscle problems, and ligament injury $(1,2)$. It is estimated that approximately one out of every four people has chronic low back pain and that it recurs at certain intervals (3).

With the use of ultrasound elastography (USE) in the musculoskeletal system over the last decade, promising results have been achieved in the diagnosis and management of muscle disorders $(4,5)$. The working principle of USE in muscle evaluation is based on the testing methods utilized to identify the mechanical features of tissues. Meaning that the deformation caused by an outside force is recorded by the measurement of temporal shift in ultrasound (US) echo $(6,7)$. However, strain elastography is operator-dependent and provides semi-quantitative values; conversely, shear-wave elastography (SWE) is considered to be a quantitative method that yields objective results. There are three different methods of SWE: transient elastography (TE), two-dimensional (2D) SWE, and point (p-) SWE. In TE, there are no B-mode anatomical images, while the other two methods can provide better data. In 2D-SWE, tissue stiffness can be determined via multiple measurements on 2D colored velocity maps of regions of interests (ROIs) determined by the operator. The third method, p-SWE, allows for multiple measurements on B-mode US images from the same area with a fixed $\mathrm{ROI}(8,9)$.

The suitability of SWE analysis in muscle tissue has been demonstrated previously $(10,11)$. Moreover, studies

Address for Correspondence: Tuba Selcuk Can

University of Health Sciences Turkey, İstanbul Haseki Training and Research Hospital, Clinic of Radiology, Istanbul, Turkey

Phone: +90 5327843477 E-mail: drtubas@gmail.com ORCID: orcid.org/0000-0002-2388-1715

Received: 04.08.2021 Accepted: 06.12.2021

Copyright 2022 by The Medical Bulletin of Istanbul Haseki Training and Research Hospital The Medical Bulletin of Haseki published by Galenos Yayinevi. 
by Murillo et al. (12) and Alis et al. (13) have utilized SWE to assess the multifidus muscle in patients with low back pain and lumbar disc herniation, respectively. There are no studies that have applied two SWE methods to evaluate the multifidus muscle in patients with lumbar disc herniation. In this study, we aimed to compare multifidus muscle elasticity on the herniated-side and non-herniated side of patients with unilateral lumbar disc herniation by using the 2D-SWE and p-SWE techniques and to compare the results obtained with each technique.

\section{Methods}

\section{Ethical Consideration}

Approval was received from the Ethics Committee of University of Health Sciences Turkey, Istanbul Haseki Training and Research Hospital, (no: 2019-38, date: 27.11.2019). After providing verbal information concerning the conduct of the study, written informed consent forms were signed by each volunteer patient.

\section{Study Design and Patient Population}

This cross-sectional study was carried out by examining the hospital records of patients who underwent lumbar magnetic resonance imaging (MRI) in July 2019. We included 22 patients who were found to have a unilateral paramedian (posterolateral) hernia at a single level through lumbar MRI. All subjects that underwent MRI had been admitted with lower back pain complaints and were found to have one-sided compression-related pain, suggesting a preliminary diagnosis of lumbar disc herniation. Patients with multiple hernias or bilateral hernias, spondylolisthesis, and spinal canal stenosis, as well as those with a history of trauma, rheumatologic disease, malignancy, infection, or back surgery, were excluded from the study.

\section{MRI}

All lumbar MRI images were performed according to the standard lumbar MRI protocol (axial T2-weighted fast spinecho; TR: 3000-4000, TE: 100, sagittal T2-weighted fast spin-echo; TR: 2500-3000, TE: 100, Sagittal T1-weighted spin-echo TR: 500, TE: 10) with a 1.5 Tesla Philips Achieva MRI device (Philips Healthcare, Best, The Netherlands). The images were evaluated at a workstation (SYNAPSE PACS, Fujifilm Medical Systems, U.S.A.) by a single radiologist with 7 years of spinal imaging experience. The observer first detected the presence of hernia from sagittal T2W images and then confirmed the presence of hernia on axial T2W images. Focal bulging of the intervertebral disc towards the lateral recess was considered a paramedian hernia (14). In the cases of nerve root contact in the lateral recess, compression and deviation in the nerve root were considered as the presence of nerve root contact in the relevant segment, and patients without nerve root contact were excluded from the study at this stage (15). Furthermore, the multifidus muscle areas were determined on both sides by drawing muscle borders with a free-hand $\mathrm{ROI}$ (Figure 1).

\section{SWE}

Two radiologists, different from the one that conducted MRI analysis, who had 12 years of experience with US and 4 years of experience with USE performed USE evaluations. The device used for SWE was an Esaote MyLab 9 device equipped with QElaXto 2D and QElaXto-pSWE with an L4-15 MHz linear probe. The evaluation of SWE was carried out in the following manner: before beginning the

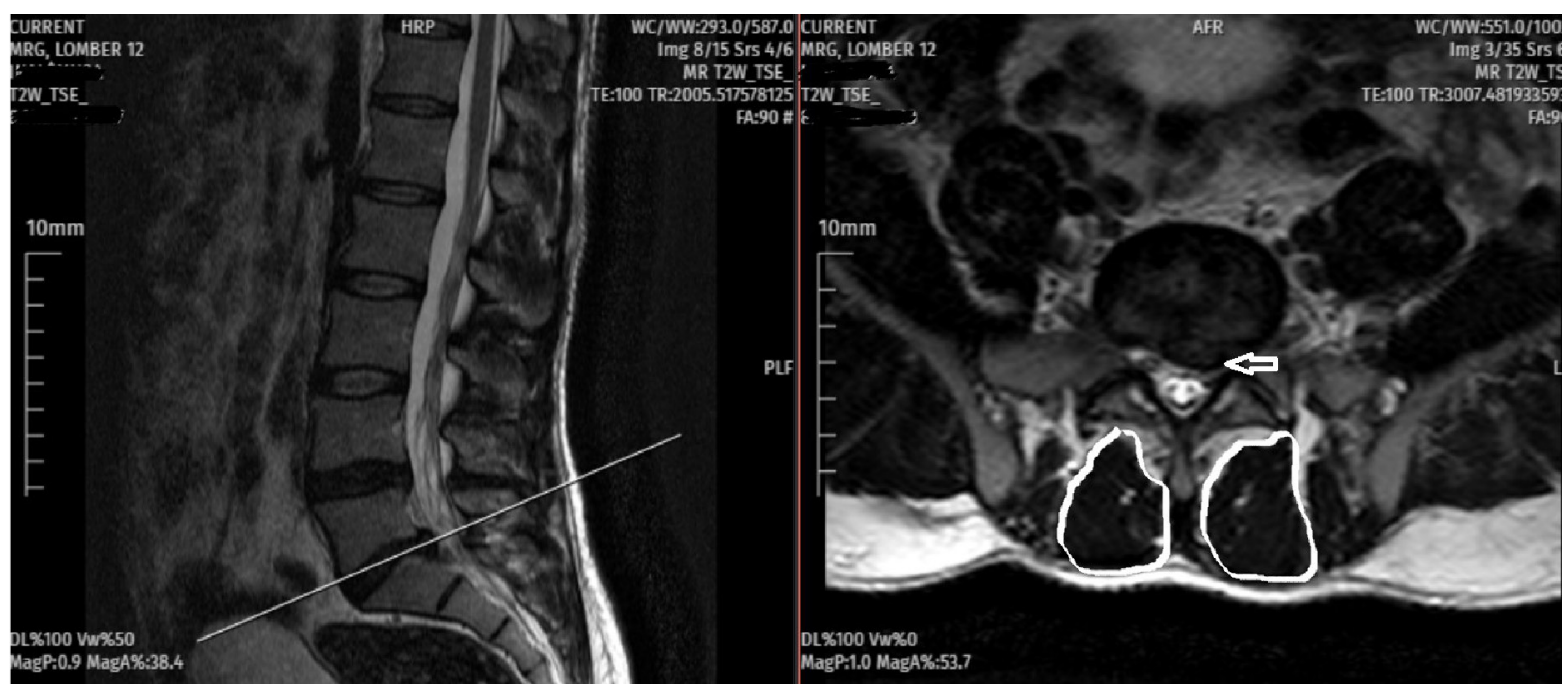

Figure 1. Sagittal T2W images are shown on the left and axial T2W images are shown on the right. A left paramedian hernia is seen at the level of L5-S1 in the axial view (arrow). Left S1 nerve compression is present in the left lateral recess. At this level, the borders of the bilateral multifidus muscle were drawn 
procedure, the first radiologist was immediately informed about the hernia level, but not the side of the hernia. The patients were brought into the prone position with their hands supporting the head in the forehead area and arms in approximately $120^{\circ}$ of abduction at normal room temperature $\left(24^{\circ} \mathrm{C}\right)(16-19)$. Rolled towels were placed under the abdominal area (to reduce lumbar lordosis) and under the ankles (for support) (16-19). The SWE measurement was performed appropriately for each level. For instance, in the presence of disc herniation at the L4$L 5$ level, the $L 5$ vertebra spinous process was identified in B-mode and the probe was shifted $2 \mathrm{~cm}$ laterally for imaging of the multifidus muscle. The probe was then switched from longitudinal orientation to transverse orientation, and the probe was rotated about $10^{\circ}$ (with the superior section moving medially) to enable a parallel position to muscle fibers (13). For the 2D-SWE measurement, a colorcoded map was created by pressing the related button. Tissue stiffness was coded with colors varying between red and blue (high to low). After this stage, the patient was warned not to breathe deeply. A total of 3 ROls, each with a diameter of $7 \mathrm{~mm}$ (13), were placed at different points in the color-coded area, followed by 2D-SWE measurements. After completing the measurements, the radiologist left the room and was replaced by the second radiologist who would carry out the p-SWE measurements. The second radiologist entered the room when the patient was lying down in the same position, and without changing the position of the patient, brought the probe into the appropriate position at the hernia level that he had been informed about immediately before entering the room. After necessary adjustments to confirm positioning, the radiologist performed 5 consecutive measurements in the image area with the fixed ROI by selecting the "point SWE (QElaXto)" mode on the device (Figure 2).

\section{Statistical Analysis}

A statistically significant difference in effect size difference (effect size $d z=0.80$ ) in the dependent group sampling was predicted to be $95 \%$. With power analysis, the total number of subjects necessary for comparison was determined to be 19 patients, for an alpha error of 0.05 (13).

The Statistical Package for Social Sciences software, version 15.0, was used for statistical analysis in the evaluation of all the data obtained from the study. Descriptive statistical results (mean, standard deviation, minimum, maximum, frequency, percentage) were reported with regard to the quantitative and qualitative characteristics of each variable. The Mann-Whitney $U$ test was used for the analyses comparing the means between two groups, the Wilcoxon test was implemented for rightleft comparisons, and the Pearson correlation coefficient was calculated to assess relationships between variables. The results were accepted and evaluated at a confidence interval of $95 \%$ and significance was identified as $p<0.05$.

\section{Results}

Twenty-two patients with unilateral lumbar disc herniation were included in the study. The age of the patients ranged between 32 and 52 years, and the mean age was $44.23 \pm 5.49$ years. Twelve patients (54.5\%) were female, and 10 patients (45.5\%) were male. With respect to age and body mass index (BMI), there were no statistically significant differences between females and males. The patients' mean height was found to be $1.69 \pm 0.11 \mathrm{~m}$ (range: 1.52-1.90 m), their mean weight

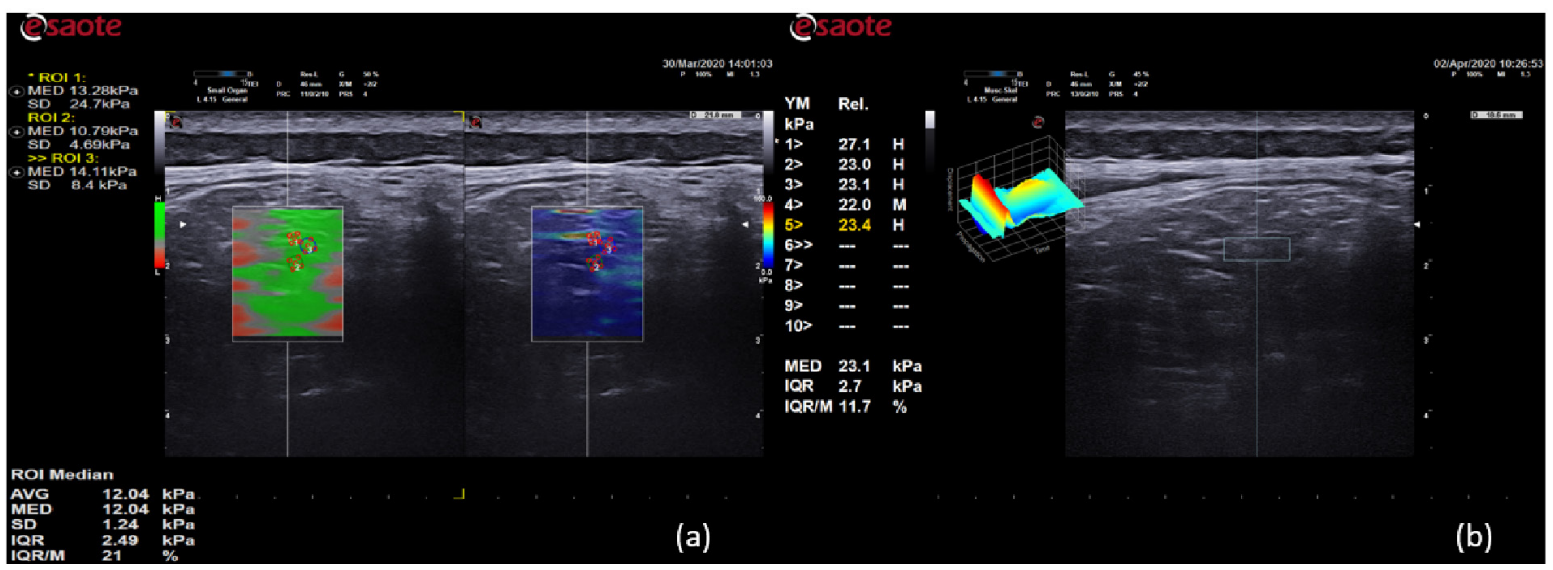

Figure 2. Elastography images, a: 2D-SWE measurement sample. This image consists of two images and is known as a "confidence map" with a green background, which shows that the measurement was made at the right place. It was necessary to do it from where it is homogeneous. The blue next to it shows three different measurements made from the field that we confirmed from the ie confidence map b: point-SWE measurement. The grayscale shows five different measurements made from the area in the ROI that we placed in the anatomical area we determined on the US image US: Ultrasound, SWE: Shear-wave elastography, ROI: Regions of interest 
Table 1. Comparison of p-SWE and 2D-SWE values on the hernia side and opposite side

\begin{tabular}{|c|c|c|c|c|c|}
\hline & \multicolumn{2}{|l|}{ Hernia side } & \multicolumn{2}{|c|}{ Non-hernia side } & \multirow[t]{2}{*}{ p-value* } \\
\hline & Mean \pm SD & $\begin{array}{l}\text { Median } \\
\text { (min-max) }\end{array}$ & Mean \pm SD & $\begin{array}{l}\text { Median } \\
\text { (min-max) }\end{array}$ & \\
\hline p-SWE (kPa) & $8.5 \pm 3.9$ & $7.7(4.6-21.4)$ & $11.6 \pm 3.3$ & $11.3(6.3-21.2)$ & 0.002 \\
\hline 2D-SWE (kPa) & $10.7 \pm 6.9$ & $8.5(3.1-28.2)$ & $13 \pm 6.8$ & $11.6(3.2-33.6)$ & 0.020 \\
\hline p-SWE vs. 2D-SWE p-value* & \multicolumn{2}{|l|}{0.239} & \multicolumn{2}{|l|}{0.016} & \\
\hline
\end{tabular}

*Related-samples Wilcoxon signed-rank test: It shows difference between the herniated and non-herniated sides of the same cases

SD: Standard deviation, min: Minimum, max: Maximum, SWE: Shear-wave elastography

Table 2. The results of p-SWE and 2D-SWE according to BMI category

\begin{tabular}{|c|c|c|c|c|c|}
\hline & \multicolumn{2}{|c|}{ Normoweight $(\mathrm{BMI}<25)$} & \multicolumn{2}{|c|}{ Overweight (BMI $\geq 25)$} & \multirow[t]{2}{*}{ p-value ${ }^{*}$} \\
\hline & Mean \pm SD & $\begin{array}{l}\text { Median } \\
\text { (min-max) }\end{array}$ & Mean \pm SD & $\begin{array}{l}\text { Median } \\
\text { (min-max) }\end{array}$ & \\
\hline Hernia p-SWE (kPa) & $10.7 \pm 4.8$ & $9.8(4.6-21.4)$ & $6.7 \pm 1.5$ & $6.3(5-9.2)$ & 0.014 \\
\hline Non-hernia p-SWE (kPa) & $13.1 \pm 3.7$ & $12.4(8.5-21.2)$ & $10.3 \pm 2.2$ & $10.7(6.3-13.5)$ & 0.059 \\
\hline Hernia 2D-SWE (kPa) & $13.7 \pm 8.8$ & $13.1(3.3-28.2)$ & $8.3 \pm 3.9$ & $8.1(3.1-17.2)$ & 0.180 \\
\hline Non-hernia 2D-SWE (kPa) & $15.1 \pm 9.8$ & $12.6(3.2-33.6)$ & $11.4 \pm 2.3$ & $11.4(7.2-16.2)$ & 0.418 \\
\hline
\end{tabular}

Table 3. Linear regression results showing parameters that were independently influential on different measurements of multifidus muscle stiffness

\begin{tabular}{|l|l|l|l|l|}
\hline & Age & Weight & Height & BMI \\
\hline Hernia p-SWE & $B=-0.552, P=0.008$ & $B=-0.648, P=0.043$ & $B=0.751, P=0.019$ & NS \\
\hline Non-hernia p-SWE & $B=-0.651, P=0.007$ & $B=-0.454, P=0.038$ & NS & NS \\
\hline Hernia 2D-SWE & $B=-0.598, P=0.004$ & NS & NS & NS \\
\hline Non-hernia 2D-SWE & $B=-0.688, P=0.002$ & NS & NS & NS \\
\hline NS: Not significant, BMI: Body mass index, SWE: Shear-wave elastography & & \\
\hline
\end{tabular}

was $71.91 \pm 10.14 \mathrm{~kg}$ (range: $50-92 \mathrm{~kg}$ ), and their mean BMI was $25.25 \pm 2.27 \mathrm{~kg} / \mathrm{m}^{2}$ (range: $20.03-29.69 \mathrm{~kg} / \mathrm{m}^{2}$ ).

The level of hernia was L3-L4 in 13.6\% ( $n=3)$, L4-L5 in $31.8 \%(n=7)$ and L5-S1 in $54.5 \%(n=12)$ of the subjects. The side of the hernia was distributed 50:50 between right and left. In terms of age and BMI, no statistically significant difference was found between those with a hernia on the right side and those with a hernia on the left side.

The p-SWE and 2D-SWE values were found to be significantly lower on the hernia side compared to the non-hernia side ( $p=0.002$ and $p=0.020$, respectively) (Table 1). Although the p-SWE and 2D-SWE results were in agreement for the hernia side $(p=0.239)$, there was a significant difference between the two tests in terms of the results obtained for the non-hernia side $(p=0.016)$. Correlation analyses confirmed this, revealing a significant relationship on the hernia side $(r=0.811, P<0.001)$ but no correlation on the non-hernia side $(p=0.078)$. The crosssectional areas of the multifidus muscle on the hernia side were found to be significantly smaller compared to the non-hernia side $(p=0.002)$.
The results showed that the hernia-side p-SWE values were significantly lower among overweight subjects $(n=12)$ compared to normoweight subjects $(n=10)$ $(p=0,014)$ (Table 2$)$.

Finally, we performed linear regression to assess the effects of various variables on the results obtained from the tests. All four parameters of muscle stiffness were defined as dependent variables in separate regression analyses, with age, weight, height, and BMI included as factors. In all analyses, age was identified as a significant factor. Weight was identified as a significant factor for both p-SWE results, while height was only significant for hernia-side $\mathrm{p}$-SWE results. Interestingly, the BMI value was not significant for any of the measurements (Table 3).

\section{Discussion}

Our results with two SWE techniques show that multifidus muscle stiffness is significantly decreased on the hernia side (compared to the non-hernia side) in patients with unilateral lumbar disc herniation. The comparison of the two techniques showed that p-SWE may result in lower stiffness values compared to 2D-SWE on the 
side of the hernia. The results showed that age was a significant parameter that affected measurement results for both sides in both methods, whereas weight was only significant for $p$-SWE results. These results suggest that age should always be considered when performing multifidus stiffness evaluation, while weight should be taken into account when using p-SWE. It is also remarkable that BMI was not found to be effective on stiffness results.

In their MRI evaluation of L5 radiculopathy, Campbell et al. (17) described isolated atrophy and fatty change in the multifidus muscle on the ipsilateral side at the L 5 level. In the following years, the multifidus muscle drew the attention of researchers since it had unisegmental innervation from a single root compared to multisegmental innervation in other paraspinal muscles. Studies were conducted on the cross-sectional area and fatty degeneration of the multifidus muscle in patients with radiculopathy (18-20). As a result of denervation, Hyun et al. (18) discovered muscle atrophy and a decrease in the cross-sectional area of the multifidus muscle. However, Battié et al. (19) discovered a larger fatty change in the hernia-side muscle, with a greater cross-sectional area than the non-hernia side. Although this difference can partially be explained by the fact that the duration of symptoms in the study of Battié et al. (19) was shorter compared to other studies, this finding still leads to questions regarding the use of multifidus muscle atrophy as a criterion in radiculopathy evaluation.

With the use of US elastography, SWE has drawn the attention of authors researching this topic due to its ability to provide quantitative data about muscle stiffness in addition to the changes in cross-sectional area and tissue structure. In a study where Creze et al. (1) evaluated SWE features at the L3 level of the paraspinal muscles in asymptomatic volunteers, in vivo results of $5.4 \pm 1.6 \mathrm{kPa}$ and ex vivo results of $5.1 \pm 1.7 \mathrm{kPa}$ stiffness were obtained for the multifidus muscle (1). In a feasibility study with 10 asymptomatic volunteers, Moreau et al. (21) evaluated the SWE features of the multifidus muscle at two different levels (L2-L3 and L4-L5), in the "passive stretching" position and at "rest". Although they worked with a small number of volunteers, they obtained intraclass correlation coefficient (ICC) values of 0.94 and 0.95 at the L2-L3 level in both positions with three different observers (in rest and passive stretching positions, respectively). They obtained relatively lower ICC values at the L4-L5 level. However, the small difference between repeatability and reproducibility and its independence from the observer were promising for the reliability of the technique. Besides these studies, Koppenhaver et al. (16) evaluated the multifidus muscle in a larger series consisting of 36 patients at three different contraction levels in addition to the rest position. At rest, multifidus SWE values were about $6 \mathrm{kPa}$ and were shown to increase in direct proportion with the amount of contraction. Sadeghi et al. (22) obtained stiffness values of $16.15,27.28$ and $45.02 \mathrm{kPa}$, respectively, in their study evaluating the SWE features of the multifidus muscle in the prone position, standing and with one arm raised. In our study, the multifidus muscle measurements in the prone position ranged between 11.30 and $11.89 \mathrm{kPa}$ on the non-hernia side. In contrast to the measurements made at one level in the literature, measurements were made at three different levels in our study, and the current differences shown may result from this feature, as well as the difference between the USE devices used. Among the few studies on symptomatic patients, Murillo et al. (12) found higher stiffness values (at the L3 level) in those with low back pain compared to controls. A similar finding was reported by Masaki et al. (23) who performed measurements at the L4 level. Koppenhaver et al. (16) also showed that multifidus stiffness was increased in people with low back pain. In these studies, which were carried out on patients with low back pain without any specific diagnosis, the finding of high stiffness can be explained by the protective mechanism and increased reflex muscle spasm (16).

In the literature on this subject, studies on this subject have almost always been conducted with 2D-SWE. In a similar study to ours by Alis et al. (13) the SWE features of the multifidus muscle were evaluated comparatively with the opposite side in patients with a single unilateral hernia at one of the three different levels. They found a mean stiffness value of $13.70 \pm 4.05-14.08 \pm 3.57 \mathrm{kPa}$ on the affected side. In our study, the mean 2D-SWE value on the affected side was 10.7 \pm 6.9 (median: 8.5) kPa, and comparisons showed the hernia side had significantly lower values compared to the non-hernia side. They also identified a negative correlation between the duration of symptoms and multifidus stiffness (13). In addition, Wan et al. (24) found a similar correlation between the duration of symptoms and fatty change in patients with low back pain. Although the correlation between the duration of symptoms and multifidus stiffness was beyond the scope of our study, we did not include symptom duration as a parameter. We found that age was independently influential on stiffness analysis with both techniques. Although advanced age does not equate to an increased duration of symptoms in most patients, it is possible that this factor may be confounding for our regression analysis. Having said this, when we consider the difference between the affected and unaffected sides in terms of the cross-sectional area of the multifidus muscle, it seems apparent that the subjects in our study had a symptom duration that made atrophy possible. On 
the other hand, regarding the changes in muscles due to denervation, Wen et al. (25) showed a decrease in muscle stiffness arising from the increase in extracellular fluid in the early period of denervation in a rabbit model; whereas, there was an increase in stiffness in the latter period (depending on increased collagen fibers despite a decrease in the cross-sectional area of the muscle). As a result, they hypothesized that the reduction in stiffness was an early sign of denervation. Rosskopf et al. (26) in their study in patients with supraspinatus tendon rupture, they found increased shear-wave velocities in stage IV patients, which were compatible with greater fatty atrophy in MRI, despite the decrease in muscle shear wave velocities up to Goutallier stage III. Although providing some insight, histological confirmation was not performed in this study, and it is also possible that these results were associated with the injury characteristics and the fact that they had applied the probe perpendicular to the muscle fibers (unlike other studies). In our study, the smaller size of the muscle areas on the hernia side can be explained by early denervation according to the hypothesis of Wen et al. (25) However, more data is required to draw conclusions since symptom duration was not evaluated in our study.

\section{Study Limitations}

Our study has some limitations. First, the presence of unilateral hernia was decided according to MRI and clinical data, and there was no electromyography verification in this study. Additionally, although the cross-sectional areas of the multifidus muscle were compared between the sides with and without hernia in the patients who were included in the study, the correlation between SWE values and symptom duration could not be evaluated since the symptom duration in the patients was not investigated. The relatively small number of people is an important limitation of the study. Finally, the changes in the multifidus muscle could not be verified histopathologically. Despite these limitations, being one of the rare studies on this subject and offering an alternative to clinicians were the important strengths of the study.

\section{Conclusion}

Our results demonstrate that two different SWE methods, 2D-SWE and p-SWE, measured lower stiffness values on the hernia side in patients with lumbar disc herniation. Moreover, we discovered that the crosssectional areas of the multifidus muscle were smaller on the hernia side compared to the opposite side. It is also important to note that age and weight may be variables that independently affect multifidus stiffness in patients with unilateral lumbar disc herniation. In line with our results, we think that SWE methods have the potential for clinical utilization in the management of patients with lumbar disc herniation. Future studies that take into account the aforementioned points and limitations should be performed to assess these methods, and more comprehensive data should be obtained to determine the utility of SWE in the clinical setting.

\section{Ethics}

Ethics Committee Approval: Approval was received from the Ethics Committee of University of Health Sciences Turkey, Istanbul Haseki Training and Research Hospital, (no: 2019-38, date: 27.11.2019).

Informed Consent: Written informed consent forms were signed by each volunteer patient.

\section{Authorship Contributions}

Concept: T.S.C., B.K.Y., Data Collection or Processing: B.K.Y., S.O., Analysis or Interpretation: S.O., Investigation: T.S.C., S.O., Methodology: B.K.Y., Project Administration: T.S.C., Supervision: T.S.C., Writing: T.S.C., B.K.Y., S.O.

Conflict of Interest: No conflict of interest was declared by the authors.

Financial Disclosure: The authors declared that this study received no financial support.

\section{References}

1. Creze M, Nyangoh Timoh K, Gagey O, Rocher L, Bellin MF, Soubeyrand M. Feasibility assessment of shear wave elastography to lumbar back muscles: A Radioanatomic Study. Clin Anat 2017;30:774-80.

2. Al Qaraghli MI, De Jesus O. Lumbar Disc Herniation. Treasure Island: StatPearls Publishing; 2021.

3. Peck J, Urits I, Peoples S, et al. A Comprehensive review of over the counter treatment for chronic low back pain. Pain Ther 2021;10:69-80.

4. van Holsbeeck M, Soliman S, Van Kerkhove F, Craig J. Advanced musculoskeletal ultrasound techniques: what are the applications? AJR Am J Roentgenol 2021;216:436-45.

5. Demarco PJ. Advanced Ultrasound Applications: Elastography and Contrast-Enhanced Ultrasound. Musculoskeletal Ultrasound in Rheumatology Review: Springer; 2021. p. 529-60.

6. Brandenburg JE, Eby SF, Song P, et al. Ultrasound elastography: the new frontier in direct measurement of muscle stiffness. Arch Phys Med Rehabil 2014;95:2207-19.

7. Zúñiga LDO, López CAG, González ER. Ultrasound Elastography in the Assessment of the Stiffness of Spastic Muscles: A Systematic Review. Ultrasound Med Biol 2021;47:1448-64.

8. Kishimoto R, Kikuchi K, Koyama A, et al. Intra-and interoperator reproducibility of US point shear-wave elastography in various organs: evaluation in phantoms and healthy volunteers. Eur Radiol 2019;29:5999-6008.

9. Mackintosh S, Young A, Lee A, Sim J. Considerations in the application of two dimensional shear wave elastography in muscle. Sonography 2020;7:13-21. 
10. Saeki J, Ikezoe T, Nakamura M, Nishishita S, Ichihashi N. The reliability of shear elastic modulus measurement of the ankle plantar flexion muscles is higher at dorsiflexed position of the ankle. J Foot Ankle Res 2017;10:18.

11. Hug F, Tucker K, Gennisson JL, Tanter M, Nordez A. Elastography for Muscle Biomechanics: Toward the Estimation of Individual Muscle Force. Exerc Sport Sci Rev 2015;43:125-33.

12. Murillo C, Falla D, Rushton A, Sanderson A, Heneghan NR. Shear wave elastography investigation of multifidus stiffness in individuals with low back pain. J Electromyogr Kinesiol 2019;47:19-24.

13. Alis D, Durmaz ESM, Alis C, et al. Shear Wave Elastography of the Lumbar Multifidus Muscle in Patients With Unilateral Lumbar Disk Herniation. J Ultrasound Med 2019;38:1695-703.

14. Fardon DF, Williams AL, Dohring EJ, Murtagh FR, Gabriel Rothman SL, Sze GK. Lumbar disc nomenclature: version 2.0: Recommendations of the combined task forces of the North American Spine Society, the American Society of Spine Radiology and the American Society of Neuroradiology. Spine J 2014;14:2525-45.

15. Pfirrmann CW, Dora C, Schmid MR, Zanetti M, Hodler J, Boos N. MR image-based grading of lumbar nerve root compromise due to disk herniation: reliability study with surgical correlation. Radiology 2004;230:583-8.

16. Koppenhaver S, Kniss J, Lilley D, et al. Reliability of ultrasound shear-wave elastography in assessing low back musculature elasticity in asymptomatic individuals. J Electromyogr Kinesiol 2018;39:49-57.

17. Campbell WW, Vasconcelos O, Laine FJ. Focal atrophy of the multifidus muscle in lumbosacral radiculopathy. Muscle Nerve 1998;21:1350-3

18. Hyun JK, Lee JY, Lee SJ, Jeon JY. Asymmetric atrophy of multifidus muscle in patients with unilateral lumbosacral radiculopathy. Spine (Phila Pa 1976) 2007;32:598-602.
19. Battié MC, Niemelainen R, Gibbons LE, Dhillon S. Is level- and side-specific multifidus asymmetry a marker for lumbar disc pathology? Spine J 2012;12:932-9.

20. Kang JI, Kim SY, Kim JH, Bang H, Lee IS. The location of multifidus atrophy in patients with a single level, unilateral lumbar radiculopathy. Ann Rehabil Med 2013;37:498-504.

21. Moreau B, Vergari C, Gad H, Sandoz B, Skalli W, Laporte S. Non-invasive assessment of human multifidus muscle stiffness using ultrasound shear wave elastography: A feasibility study. Proc Inst Mech Eng H 2016;230:809-14.

22. Sadeghi S, Quinlan K, E Eilertson K, et al. Changes in Shear Modulus of the Lumbar Multifidus Muscle during Different Body Positions. J Biomech Eng 2019.

23. Masaki M, Aoyama T, Murakami T, et al. Association of low back pain with muscle stiffness and muscle mass of the lumbar back muscles, and sagittal spinal alignment in young and middle-aged medical workers. Clin Biomech (Bristol,Avon) 2017;49:128-33.

24. Wan Q, Lin C, Li X, Zeng W, Ma C. MRI assessment of paraspinal muscles in patients with acute and chronic unilateral low back pain. Br J Radiol 2015;88:20140546.

25. Wen J, Wang $Y$, Jiang W, et al. Quantitative Evaluation of Denervated Muscle Atrophy with Shear Wave Ultrasound Elastography and a Comparison with the Histopathologic Parameters in an Animal Model. Ultrasound Med Biol 2018;44:458-66.

26. Rosskopf AB, Ehrmann C, Buck FM, Gerber C, Flück $M$, Pfirrmann CW. Quantitative Shear-Wave US Elastography of the Supraspinatus Muscle: Reliability of the Method and Relation to Tendon Integrity and Muscle Quality. Radiology 2016;278:465-74. 